\title{
REMOTE CONTROL OF RAILWAY SWITCH HEATING USING GSM MODEMS
}

\author{
Alexandru POPOV, Dragoș-Vasile BRATU, Sorin-Aurel MORARU \\ Transylvania University of Braşov, Dept. of Automatics and Technology of Information, Romania \\ e-mail: alexandru.popov@unitbv.ro,dragos.bratu@unitbv.ro, smoraru@unitbv.ro
}

\begin{abstract}
This research activity examines the possibility of remote control of the heating of railway switches. The study and implementation of the remote-control system of resistive heaters using the Global Mobile Communication System (GSM) through SMS messages (Short Message Service) and AT (attention) commands is presented in this article. The research works envisaged are convergent on the functionality of a GSM protocol, which allows the user to control the heating of the remote railway switches, away from the place where the switches were installed. The notions of serial communication, electronic control and AT control have been applied to expand the system. To increase the robustness of the system, a feedback mechanism was introduced so that users will be able to get a feedback status of the system when the command was successfully sent or in the case of voltage drops and system restarts.
\end{abstract}

KEYWORDS: railway switch; resistive heaters; GSM protocol; attention command

\section{Introduction}

The crossing of the trains from one railway line to another is possible with the help of a special installation, called a track changer.

The railway switch is a component part of a rail changer, made up of point blades, sliding supports and two fixed rails. The actuation of the point blades is done by the handling device through the traction bar. For the switches to function adequately in extreme conditions (abundant snow, very low temperatures), each switch is equipped with a heating system composed of four resistive elements mounted on fixed and movable parts [1]. Figure 1 shows a switch equipped with a resistive heating system.

The switches are found at the entry points of stations for access from open lines, in railway stations for access to different lines, as well as at the exit of stations for changing the path. It can be stated that the size or limit of a station is given by the distance between the entry switches and the exit switches of the station. Figure 2 illustrates a railway station on double line, indicating the normal position of the switches, their numbering, as well as the distribution of the supply of the switch heaters on the power supply panels (PSP).
The command to couple or decouple the supply of the heaters is currently made by means of control keys mounted in the railway station and cables with wires that connect to the supply panels (PSP). Taking into account the high risk of control cable failure in the railway's proximity, alternative means for transmitting this command are proposed [2]. A solution proposed for solving this problem is described in the present paper and consists of the transmission of the command using GSM support [3].

\section{Functional Description}

The command to couple or decouple the switch heaters is sent from a mobile terminal as a written message (for example: 01 for ON and 10 for OFF), it is then received by a GSM modem mounted in the power supply box near the switches, and delivered to the remote control system (SCD) in the form of an electrical signal thus ordering the coupling or uncoupling of the power contacts which supply the PSPs. After command execution, a confirmation message is received on the phone. Figure 3 shows the control scheme of the resistive heater remote control through the GSM network [3, 4].

The components of the SCD assembly are: an 8bit microcontroller, a global system for mobile (also 
known as GSM) modem, a voltage regulator, an RS232 Serial Communication module and some other electronic parts such as optocoupler, diodes, transistor, capacitors and a $12 \mathrm{v}$ relay, mounted on a wiring made in Express PCB and represented in Figure $4[4,7]$.

The microcontroller is of type PIC16F887 and it is a 40-pin and 8-bit architecture with nanoWatt technology. The price for it is economical and the architecture is user-friendly so it can be easy to use and to configure. The task of the microcontroller is to accomplish the communication, using the GSM Maestro 100 modem through the serial port at a speed of 300 bits per second (bps) between the sender of the
SMS and the railroad switch in the end. In terms of power supply, a voltage regulator LM2575-5 of 1A is used achieving the reduction of the supply voltage from $12 \mathrm{~V}$ continuous to $5 \mathrm{~V}$ continuous required for PIC microcontroller and MAX232IN circuit. A 330 $\mu \mathrm{H}$ coil is used which together with the voltage regulator above mention makes a steady power supply distribution.

The MAX232IN converter is an integrated circuit which makes the transformation from logical signals $(0 / 1$ logic $)$ to $\mathrm{RS} 232$ signals $(+8 /-8 \mathrm{~V})$ required for communication with the GSM modem $[4,5]$.

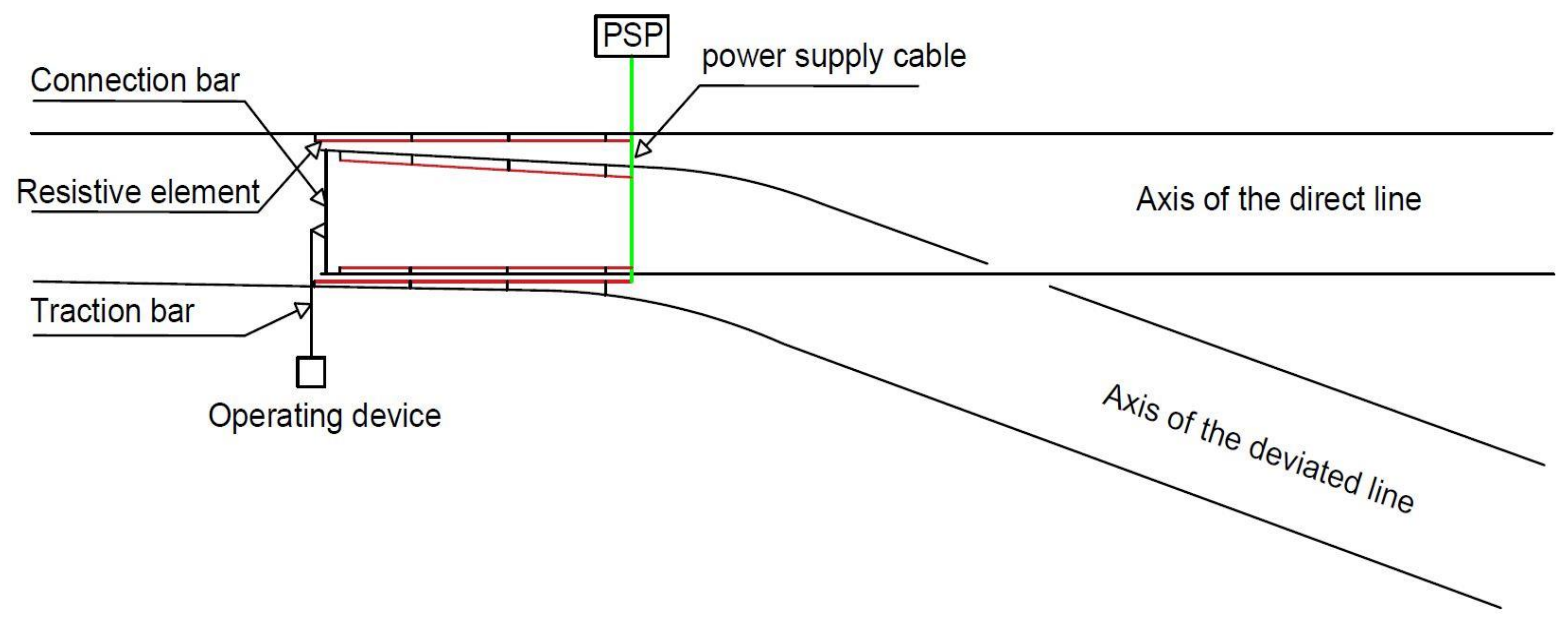

Fig. 1. Diagram of a switch equipped with a resistive heating system

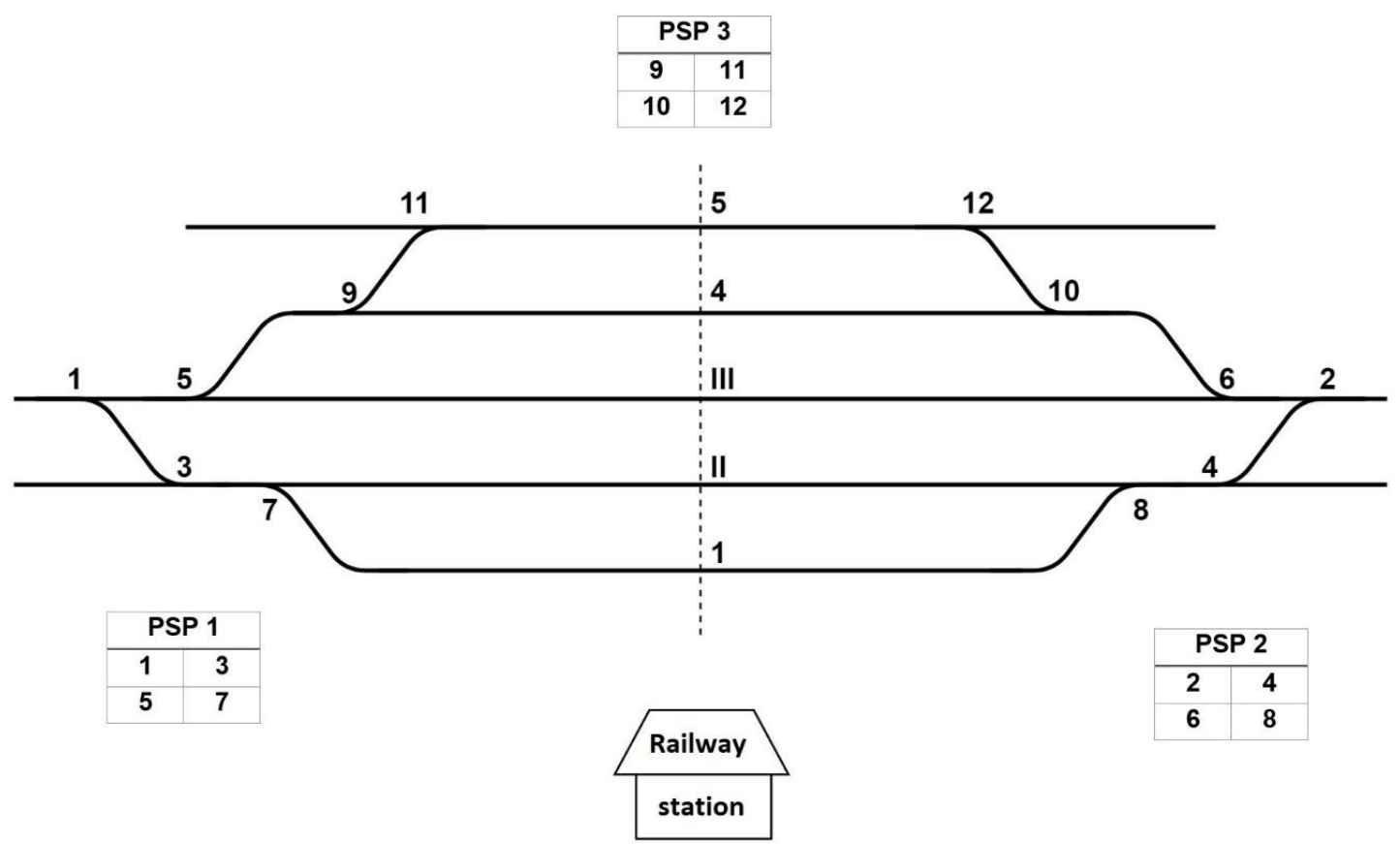

Fig. 2. Schematic of a double-track railway station, switch position and heater power supply position on the panels 


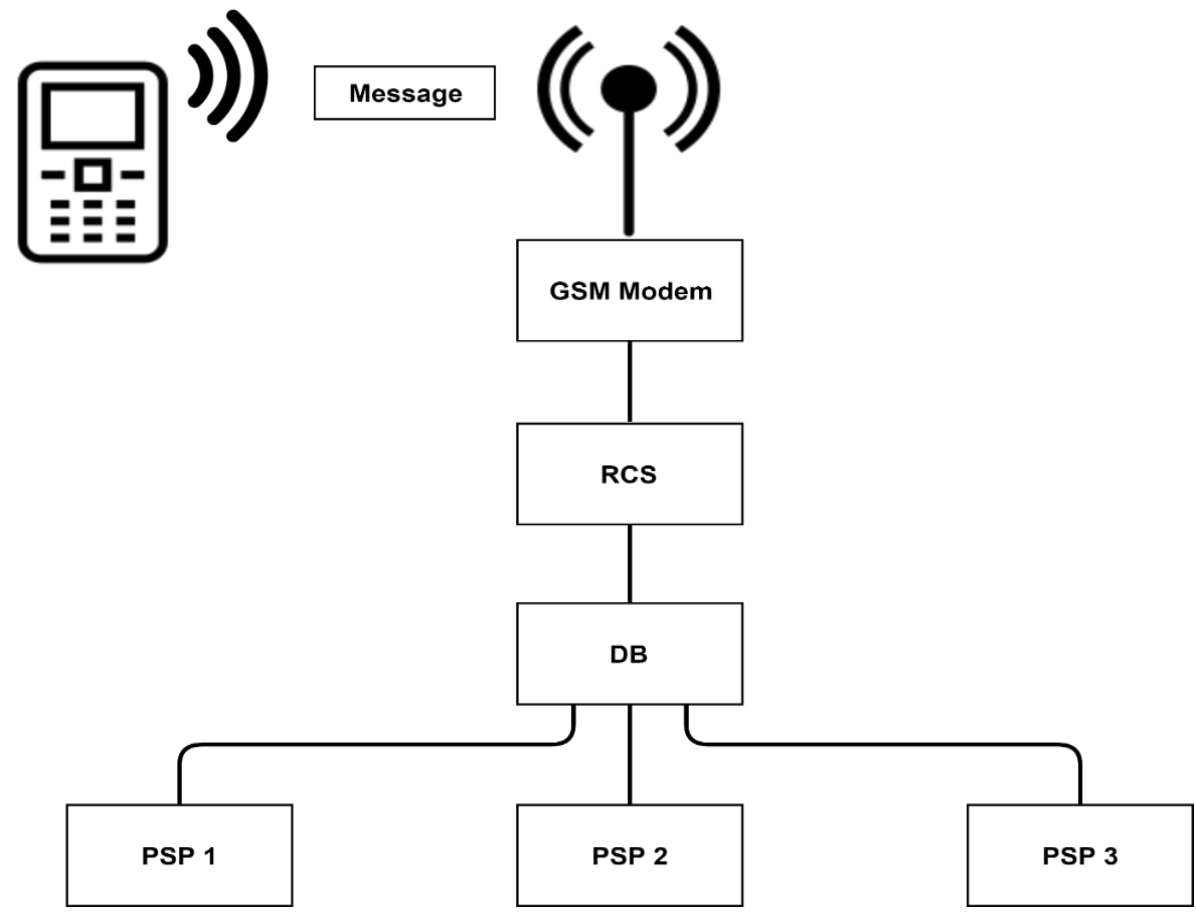

Fig. 3. Diagram of the command system of resistive heating of railway switches using GSM networking

Besides that, for better protection, the LTV825 optocoupler is used that performs the intermediation between the logic levels of control (0/1) from the microcontroller and the final command transistor BD139. The voltage level that passes through the transistor is a continuous voltage level of $12 \mathrm{~V}$ and a maximum 1.5 A. This signal is used for the railroad switch relay control, and this relay will command a voltage level of $220 \mathrm{~V}$ AC used for power contactor. The $1 \mathrm{~N} 4007$ diode in parallel with the $12 \mathrm{~V}$ relay coil achieves the protection of the BD139 transistor upon decoupling.

The optocoupler command is made by the microcontroller through the pin 20 (RD1 - check Figure 5) set as output. The reading of the (open / closed) state of the $12 \mathrm{~V}$ relay is done by passing the ground connection through an auxiliary contact of the relay. The presence or absence of the ground is performed at the input pin 10 (RE2 pin) of the microcontroller set as input pin. PIC16F887 communicates via MAX232 with the two-pin modem: TX for transmission, RX for receiving characters from the modem.

AT commands are instructions used to control the modem. AT stands for ATtention. Every command line starts with "AT" and then followed by the command.

The AT commands used for this system are: AT $+\mathrm{CMGD}=1,4$ for deleting all messages received in SIM card, AT + CMGS for sending SMS, AT +
CMGR for reading SMS received by modem and then read by PIC16F887 [6].

The programming of PIC16F887 was performed with the PICKit 2 programmer. The program was written in $\mathrm{C}$ language and the $\mathrm{n}$ Integrated Development Environment (IDE) used was MPLAB v8.10. The compilation of the program was done with the compiler HI-TECH PIC C Lite 9.60. The hexadecimal (.hex) file generated by the compiler was written in the program memory of PIC16F887 with the PICKit 2 programmer from MPLAB IDE v8.10 $[4,6]$.

\section{Software Description}

The software application was developed in $\mathrm{C}$ language. The main structure is as follows: In the beginning, some configurations are made such as the prescaler is set at a resolution of 8 bits for Timer 0 , then the watchdog timer timeout is configured and the used pins are configured as output or input. In order to know the last value that was set for the relay, a reading from EEPROM (Electrically Erasable Programmable Read-Only Memory) is made and the value will be restored from the already mentioned memory. If the relay is opened then the command to open back the relay will be sent. This is advantageous in the case of a voltage drop when the board is restarted because the relay will be set with the last command that was used before (Fig. 6). 


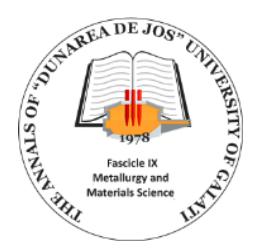

THE ANNALS OF "DUNAREA DE JOS” UNIVERSITY OF GALATI

FASCICLE IX. METALLURGY AND MATERIALS SCIENCE

№. 1 - 2019, ISSN 2668-4748; e-ISSN 2668-4756

Article DOI: $\underline{\text { https://doi.org/10.35219/mms.2019.1.07 }}$

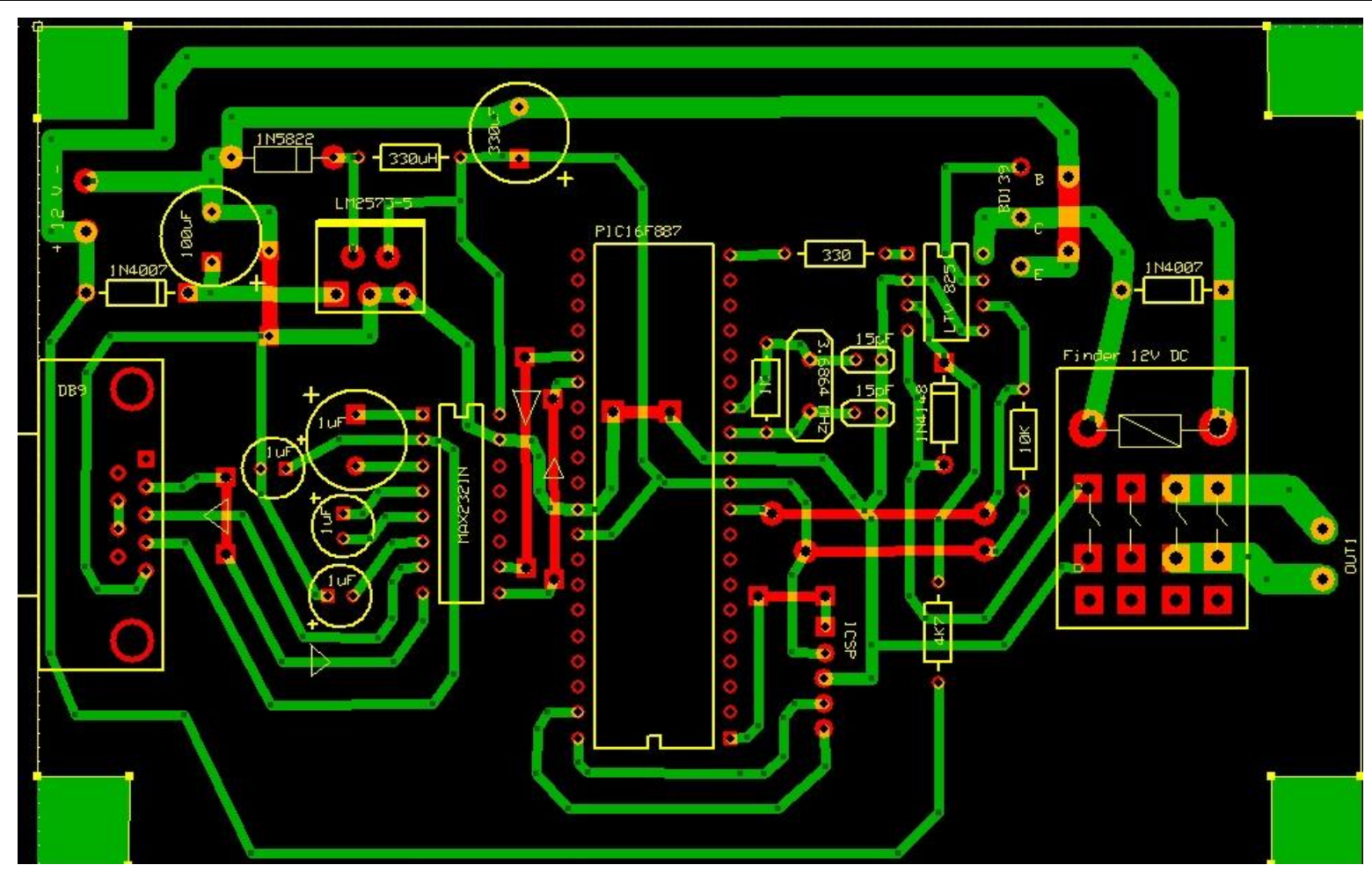

Fig. 4. RCS components assembly schematic

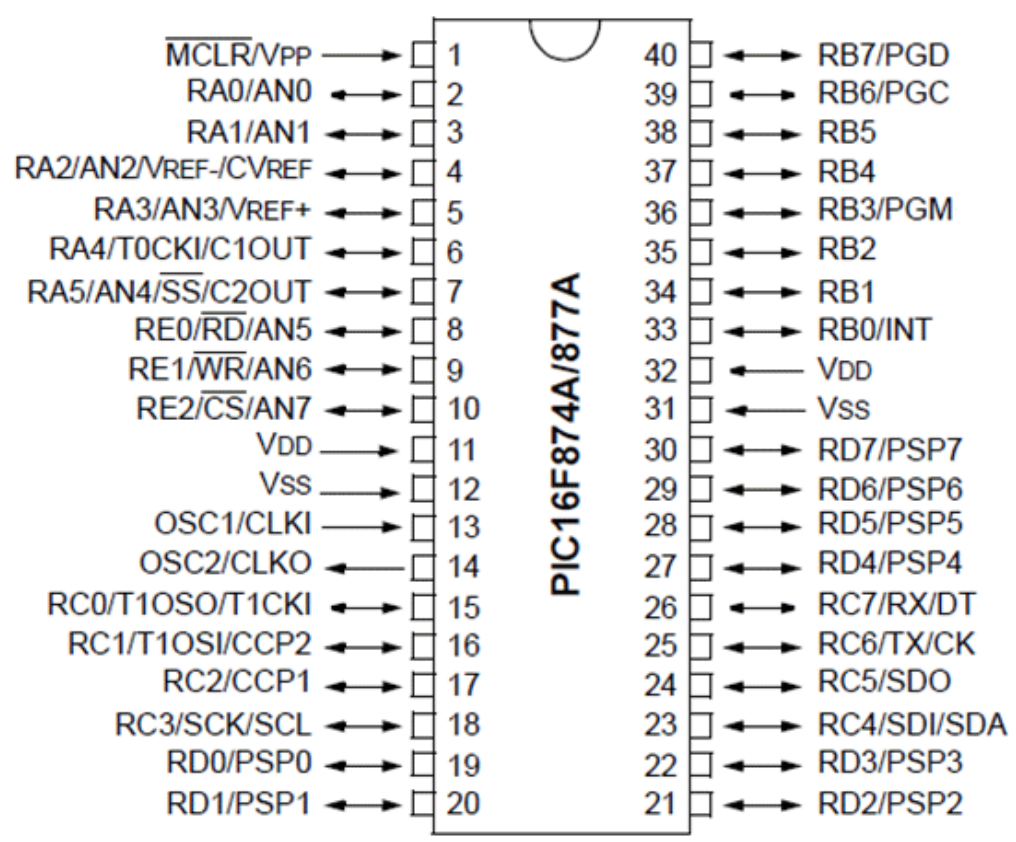

Fig. 5. PIC16F877A pin diagram (pinout) 


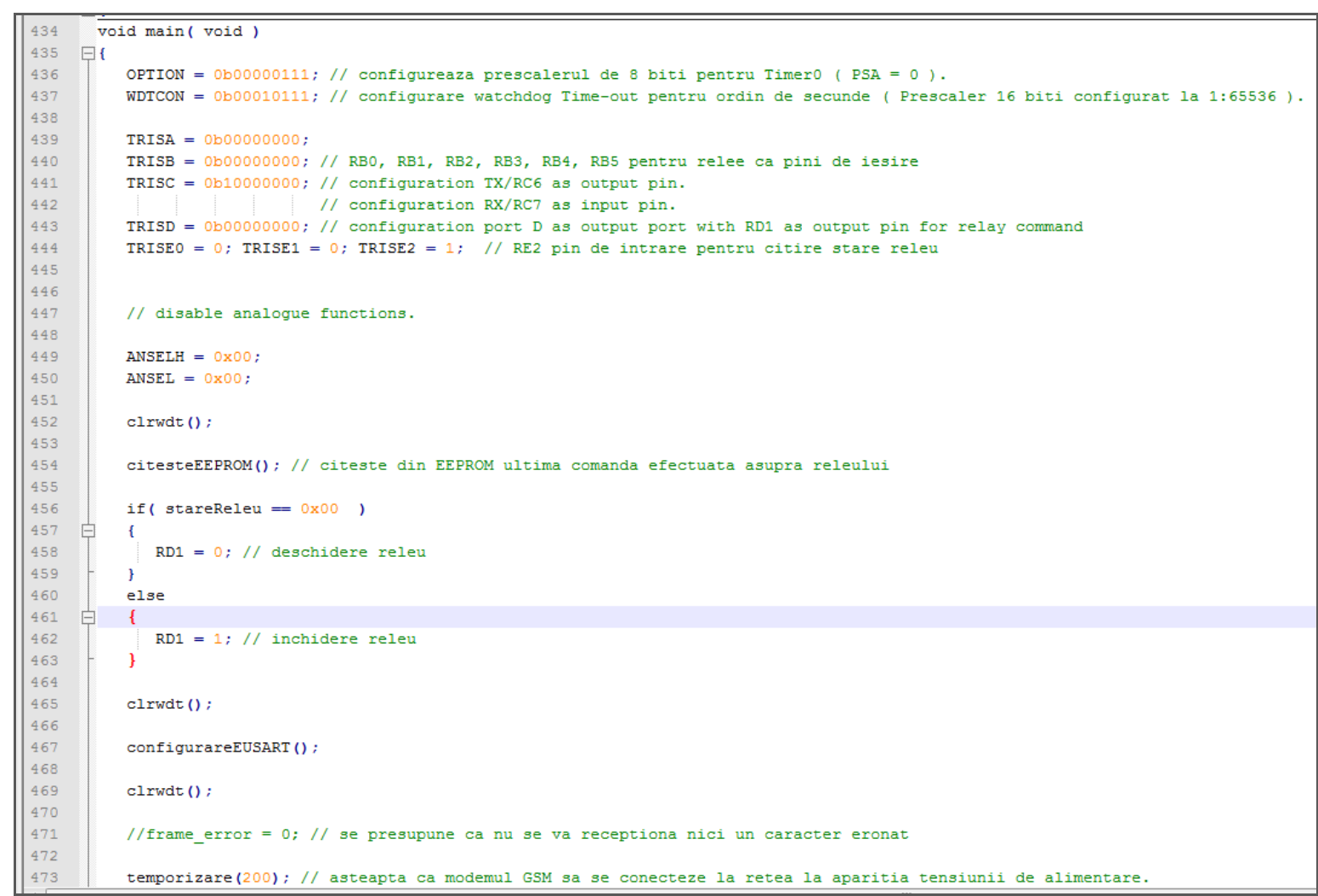

Fig. 6. Main code with configurations and fail-safe features

The next step is to configure the EUSART (Enhanced Universal Asynchronous Receiver Transceiver) module where the baud rate is configured at $300 \mathrm{bps}$ along with other configurations (frequencies, asynchronous mode, enabling the interrupts for reception).

If the power is down, the modem needs some time (almost 70 seconds) to get into connection with the nearest mobile cell tower. For that a waiting time delay of 70 seconds was introduced.

Because this fail-safe mechanism is very important, firstly a message, which specifies that a problem has been detected and a voltage drop-out has happened, is being sent alongside with the state of the relay (if it is ON or NOT) so that the user will know for sure if the data from the EEPROM is successfully restored and if it is the correct one [7].

Secondly, the code will enter a continuous loop also known as while loop that will check regularly if a SMS is received from the user. If it receives a message and it is not an accepted command it will react immediately with invalid command.

If the request is valid, firstly it will send the command to the relay via RD1 and then if the PIN is set, it will save it into the EEPROM. A feedback message will be sent confirming the command and the relay is set in the desired position.
Available commands are made up on 4 bytes buffers and they are as follows: "01ON" to turn on the relay "1OFF" to turn off the relay and for the current status of the relay the command is "STAT".

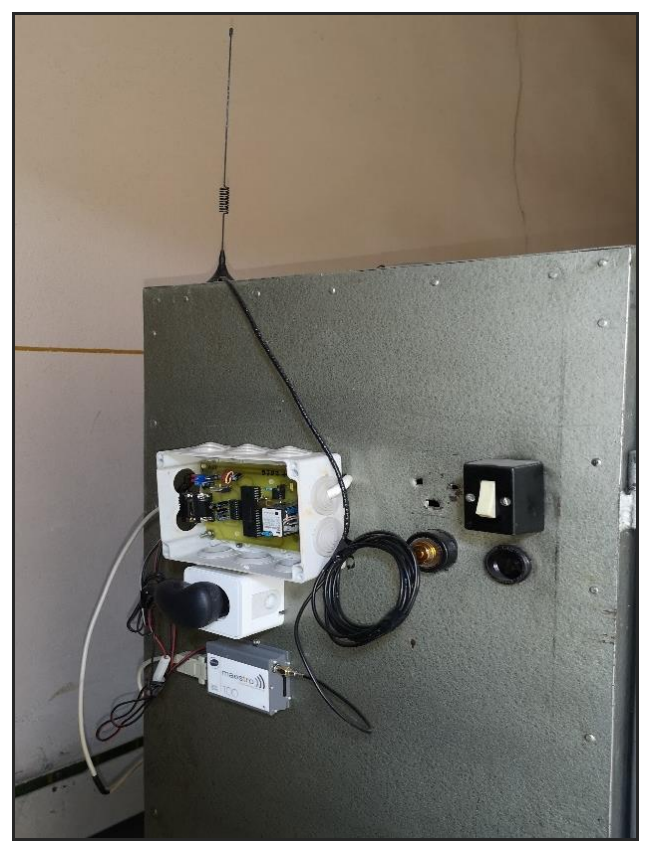

Fig. 7. Switch heating remote control device, mounted on a metal panel 
The mobile phone number where the messages can be sent, is configurable from the source code. The new code can be flashed on PIC microcontroller with the updated number.

This C-module has in total 12 functions including main function and it uses a 68 bytes buffer used for storing the message from the GSM modem alongside with some safety checks (in case the message is longer than 68 bytes the buffer will be overwritten!) [8].

\section{Conclusions}

Controlling railway switch heating elements through GSM modems eliminates the need to use control cables, and the potential failures that result from their use, which is significant, given that cable downtime results in railway switch downtime as well, impacting train traffic.

This type of equipment was designed and tested in the PRAM-TM (relay protection, automation and electrical measurement - telemechanics) laboratory in Braşov, Romania. Figure 7 illustrates the actual equipment installed and functioning.

This solution has the following advantages:

- the possibility of placing orders from a central command point;

- high accuracy and reliability;

- in the case of power failure, the system restarts executing the last received command;

- financial savings are accomplished by removing the necessity for a control cable that could reach the length of $1-2 \mathrm{~km}$;

- low cost price, resulting from technological simplicity;

- high versatility (it can be further developed by adding sensors for temperature and snow deposition);

- commands can be timed, based on the weather forecast;
- the application can be integrated into complex SCADA systems, definitively eliminating the human factor; tenfold;

- the speed of command execution is increased

- the application allows for multiple commands, by generating and sending a message to multiple areas;

- redundancy can be ensured by using multiple transfer mediums at once (such as fiber optics), thereby decreasing downtime risk in case of GSM failure.

The use of the remote-control system of the heat pumps using the GSM data transmission and reception network leads to a significant improvement in the safety of trains in extreme weather conditions during winter.

\section{References}

[1]. Golovash A. N., Esipenko V. S., Pimenov I. Y., Switch Heating Device Bibliographic data, Patent No. RU2232222 (C1)2004-07-10.

[2]. Yantang G., Electric Heating Snow Removing Device for Railway Points Bibliographic data, Patent No. CN201433383 (Y)2010-03-31.

[3]. Baruchov V. A., Kochubey V., Electrical Heating Device of Track Switches Type Seit-04 Bibliographic data, Patent No. RU2582627 (C1)-2016-04-27.

[4]. Lukman A., Olayemi M. O., Kolo J., Ajao A., Project-Based Microcontroller System Laboratory Using Bk300 Development Board with PIC16F887 Chip, International Journal of Embedded systems and Applications (IJESA), 5 (3), September 2015.

[5]. Pan Dai, GSM Remote Controler Heater, Technology and Comunication, 2014.

[6]. Parab J. S., Shelake V. G., Kamat R. K., Naik G.M., Exploring C for Microcontrollers: A Hands on Approach, 2007.

[7]. ***, ARC Electronics, RS232 Tutorial on Data Interface and cables, http://www.arcelect.com/rs232.htm/, accessed 10.10.2019. [8]. ***, Serial data communication connection over GSM data channel,

https://go.galegroup.com/ps/anonymous?id=GALE\%7CA2253160 $41 \&$ sid= $=$ googleScholar\&v=2.1\&it=r\&linkaccess=abs\&issn=17269 $679 \& \mathrm{p}=\mathrm{AONE} \& \mathrm{sw}=\mathrm{w}$. 